\title{
A MATROID VIEW OF KEY THEOREMS FOR EDGE-SWAPPING ALGORITHMS
}

\author{
JON LEE AND LEO LIBERTI
}

\begin{abstract}
We demonstrate that two key theorems of Amaldi, Liberti, Maffiolo and Maculan (2009), which they presented with rather complicated proofs, can be more easily and cleanly established using a simple and classical property of binary matroids. Besides a simpler proof, we see that both of these key results are manifestations of the same essential property.
\end{abstract}

Our goal is to demonstrate that two graph-theoretic theorems from [1] are direct manifestations of a simple and classical property of binary matroids. This follows the line of matroids sometimes serving as a means to simplify and unify combinatorial theorems involving graphs and coordinatized vector spaces.

We assume some very basic familiarity with matroid terminology and theory (see [2]), and we rely on elementary notions from graph theory. For a matroid $M$, we denote its dual matroid by $M^{*}$ (i.e., the matroid on the same ground set as $M$ but having its set of bases to be the set of complements of bases of $M)$. For $S \subset E$, we let $S^{c}$ denote the complement of $S$ in $E$. For $S, U \subset E$, we denote the symmetric difference $(S \backslash U) \cup(U \backslash S)$ by $S \Delta U$.

Let $G$ be a finite connected graph with edge set $E$. Let $T \subset E$ be a spanning tree of $G$. For an edge $f \notin T$, let $C(T, f)$ be the unique cycle of $G$ contained in $T \cup\{f\}$. Similarly, for an edge $e \in T$, let $D(T, e)$ be the unique cocycle of $G$ contained in $T^{c} \cup\{e\}$. From the point of view of matroid theory, $T$ is a base of the graphic matroid of $G$ and $T^{c}$ is a base of the cographic matroid of $G$, so $C(T, f)$ and $D(T, e)$ are fundamental circuits (in the matroid sense).

For an edge $f \notin T$ and an edge $e \in C(T, f) \backslash\{f\}, T \cup\{f\} \backslash\{e\}$ is also a spanning tree of $G$, and hence a base of the graphic matroid of $G$. Of course then, $(T \cup\{f\} \backslash\{e\})^{c}$ is also the complement of a spanning tree of $G$, so $(T \cup\{f\} \backslash\{e\})^{c}$ is a base of the cographic matroid of $G$.

With our notation, Theorem 1 of $[1$ is as follows

Theorem 1. Let $e \in T^{c}, f \in T^{c}, e \neq f, g \in C(T, e) \cap C(T, f)$. Then

$$
C(T \cup\{e\} \backslash\{g\}, f)=C(T, e) \Delta C(T, f) .
$$

The proof of Theorem 1 presented in [1 is not egregiously lengthy, but it is a bit ad hoc. However, the authors also present a Theorem 9, which they demonstrate with a long (three page) proof that is relegated to an appendix. In the way that it is stated, it is not immediately clear that the result is dual to Theorem 1 . Here, we state it in a way that makes it easier to directly compare.

Theorem 9. Let $e \in T, f \in T, e \neq f, g \notin D(T, e) \cap D(T, f)$. Then

$$
D(T \cup\{e\} \backslash\{g\}, f)=D(T, e) \Delta D(T, f) .
$$

Date: January 20, 2012. Revised March 27, 2012. 
We can easily see, now, that these theorem are just manifestations of the same phenomenon, which we state now in matroid language.

Let $B \subset E$ be a base of a matroid $M$ on ground set $E$. For $e \in B^{c}, \mathcal{C}_{M}(B, e)$ denotes the unique circuit of $M$ contained in $B \cup\{e\}$. The matroid $M$ is binary if it can be represented by a matrix over the two element field $G F(2)$.

Theorem P. Suppose that $B$ is a base of a binary matroid $M$. Let $e \in B^{c}, f \in B^{c}, e \neq f$, $g \in \mathcal{C}_{M}(B, e) \cap \mathcal{C}_{M}(B, f)$. Then

$$
\mathcal{C}_{M}(B \cup\{e\} \backslash\{g\}, f)=\mathcal{C}_{M}(B, e) \Delta \mathcal{C}_{M}(B, f) .
$$

Theorem 1 is just Theorem $\mathrm{P}$, when $M$ is the graphic matroid of $G$. Theorem 9 is just Theorem $\mathrm{P}$, when $M$ is the cographic matroid of $G$. Note that graphic and cographic matroids are binary.

It can also be useful to think of the equivalent dual re-statement of Theorem 9:

Theorem $\mathbf{P}^{*}$. Suppose that $B$ is a base of a binary matroid $M$. Let $e \in B, f \in B, e \neq f$, $g \in \mathcal{C}_{M^{*}}\left(B^{c}, e\right) \cap \mathcal{C}_{M^{*}}\left(B^{c}, f\right)$. Then

$$
\mathcal{C}_{M^{*}}\left(B^{c} \cup\{g\} \backslash\{e\}, f\right)=\mathcal{C}_{M^{*}}\left(B^{c}, e\right) \Delta \mathcal{C}_{M^{*}}\left(B^{c}, f\right) .
$$

Theorems $\mathrm{P}$ is classical and can be found, for example, as a special case of Theorem 9.1.2, part (vii) in [2, p. 304]. In the interest of making this note self contained and to demonstrate how truly elementary Theorem $\mathrm{P}$ is, we present a very short and direct proof of it. Before proceeding to the proof of Theorem $\mathrm{P}$, we need to recall basic facts concerning representations of a matroid. If $B$ is a base of a matroid $M$ that is representable over a field $\mathbb{F}$, then $M$ has a representation over $\mathbb{F}$ as a standard representative matrix

$$
A=B \quad\left[\begin{array}{l|l}
B & B^{c} \\
I & N
\end{array}\right]
$$

where here we think of $B$ and $B^{c}$ as ordered sets. Furthermore, for $e \in B$, the support of the row of $A$ indexed by $e$ is precisely $\mathcal{C}_{M^{*}}\left(B^{c}, e\right)$.

Proof. (Theorem $P$ ). We proceed by establishing the equivalent Theorem $\mathrm{P}^{*}$. The matrix $A$ has a pair of rows indexed by $e, f \in B$, and $g \in B^{c}$ is in the support of each of these two rows:

$$
e \quad\left[\begin{array}{lllllllllllllll}
0 & \ldots & 0 & 1 & 0 & 0 & \ldots & 0 & \times & \ldots & \times & 1 & \times & \ldots & \times \\
0 & \ldots & 0 & 0 & 1 & 0 & \ldots & 0 & \times & \ldots & \times & 1 & \times & \ldots & \times
\end{array}\right]
$$

Pivoting on the entry in the top row and column $g$ leads to a standard representative matrix for $M$ with respect to the base $B \cup\{e\} \backslash\{g\}$, the complement of which is $B^{c} \cup\{g\} \backslash\{e\}$. So, after the pivot, the support of the second row is precisely $\mathcal{C}_{M^{*}}\left(B^{c} \cup\{g\} \backslash\{e\}, f\right)$. Moreover, the result in the second row is the $G F(2)$ sum of the two rows, which is precisely $\mathcal{C}_{M^{*}}\left(B^{c}, e\right) \Delta \mathcal{C}_{M^{*}}\left(B^{c}, f\right)$.

\section{REFERENCES}

1. E. Amaldi, L. Liberti, F. Maffioli and N. Maculan, Edge-swapping algorithms for the minimum fundamental cycle basis problem, Mathematical Methods of Operations Research, Vol. 69 (2009) 205-223.

2. J. Oxley, "Matroid Theory,", Oxford University Press, New York, 1992.

Jon Lee: IOE Department, University of Michigan, Ann Arbor, Mi 48109-2117 USA

E-mail address: jonxlee@umich.edu

Leo Liberti: LiX, École Polytechnique, 91128 Palaiseau, France.

E-mail address: liberti@lix.polytechnique.fr 